Supplement of Atmos. Chem. Phys., 21, 3763-3775, 2021

https://doi.org/10.5194/acp-21-3763-2021-supplement

(C) Author(s) 2021. CC BY 4.0 License.

(c) (1)

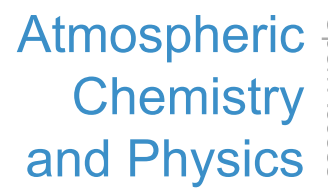

go

Supplement of

\title{
Identification and source attribution of organic compounds in ultrafine particles near Frankfurt International Airport
}

Florian Ungeheuer et al.

Correspondence to: Alexander L. Vogel (vogel@iau.uni-frankfurt.de)

The copyright of individual parts of the supplement might differ from the article licence. 
Table S1. Overview of the sampling times (only during Frankfurt Airport operating hours between 5 AM and 11 PM).

\begin{tabular}{|c|c|c|c|c|}
\hline Sample & Start & Stop & Collection time $[\mathrm{h}]$ & $\mathrm{Dp}(\mathrm{aer})[\mu \mathrm{m}]$ \\
\hline FF7 & $8 / 31 / 20195: 00$ & $8 / 31 / 201923: 00$ & 18 & $0.01-0.018$ \\
\hline FF8 & 8/31/2019 5:00 & $8 / 31 / 201923: 00$ & 18 & $0.018-0.032$ \\
\hline FF10 & 9/4/2019 5:00 & $9 / 4 / 201923: 00$ & 18 & $0.01-0.018$ \\
\hline FF11 & 9/4/2019 5:00 & 9/4/2019 23:00 & 18 & $0.018-0.032$ \\
\hline FF13 & 9/6/2019 12:00 & 9/7/2019 23:00 & 29 & $0.01-0.018$ \\
\hline FF14 & 9/6/2019 12:00 & 9/7/2019 23:00 & 29 & $0.018-0.032$ \\
\hline FF15 & 9/6/2019 12:00 & 9/7/2019 23:00 & 29 & $0.032-0.056$ \\
\hline FF16 & $9 / 11 / 2019$ 10:00 & $9 / 12 / 201923: 00$ & 31 & $0.01-0.018$ \\
\hline FF17 & 9/11/2019 10:00 & $9 / 12 / 201923: 00$ & 31 & $0.018-0.032$ \\
\hline FF18 & 9/11/2019 10:00 & 9/12/2019 23:00 & 31 & $0.032-0.056$ \\
\hline FF19 & 9/15/2019 9:00 & $9 / 16 / 201923: 00$ & 32 & $0.01-0.018$ \\
\hline FF20 & 9/15/2019 9:00 & $9 / 16 / 201923: 00$ & 32 & $0.018-0.032$ \\
\hline FF21 & 9/15/2019 9:00 & 9/16/2019 23:00 & 32 & $0.032-0.056$ \\
\hline Blank & $9 / 18 / 2019$ 17:00 & $9 / 23 / 201911: 30$ & - & $0.01-0.018$ \\
\hline Blank & 9/18/2019 17:00 & $9 / 23 / 201911: 30$ & - & $0.018-0.032$ \\
\hline Blank & $9 / 18 / 2019$ 17:00 & $9 / 23 / 201911: 30$ & - & $0.032-0.056$ \\
\hline FF28 & $9 / 27 / 201915: 40$ & 9/30/2019 11:00 & 49 & $0.01-0.018$ \\
\hline FF29 & $9 / 27 / 2019$ 15:40 & 9/30/2019 11:00 & 49 & $0.018-0.032$ \\
\hline FF30 & $9 / 27 / 2019$ 15:40 & 9/30/2019 11:00 & 49 & $0.032-0.056$ \\
\hline FF31 & $9 / 30 / 201912: 05$ & $10 / 1 / 201923: 00$ & 29 & $0.01-0.018$ \\
\hline FF32 & 9/30/2019 12:05 & $10 / 1 / 201923: 00$ & 29 & $0.018-0.032$ \\
\hline FF33 & 9/30/2019 12:05 & $10 / 1 / 201923: 00$ & 29 & $0.032-0.056$ \\
\hline FF43 & $10 / 16 / 20195: 00$ & 10/18/2019 23:00 & 54 & $0.01-0.018$ \\
\hline FF44 & 10/16/2019 5:00 & 10/18/2019 23:00 & 54 & $0.018-0.032$ \\
\hline FF45 & $10 / 16 / 20195: 00$ & $10 / 18 / 201923: 00$ & 54 & $0.032-0.056$ \\
\hline
\end{tabular}


Table S2. Most prominent jet engine lubrication oil constituents and the thermal decomposition product TMP-P.

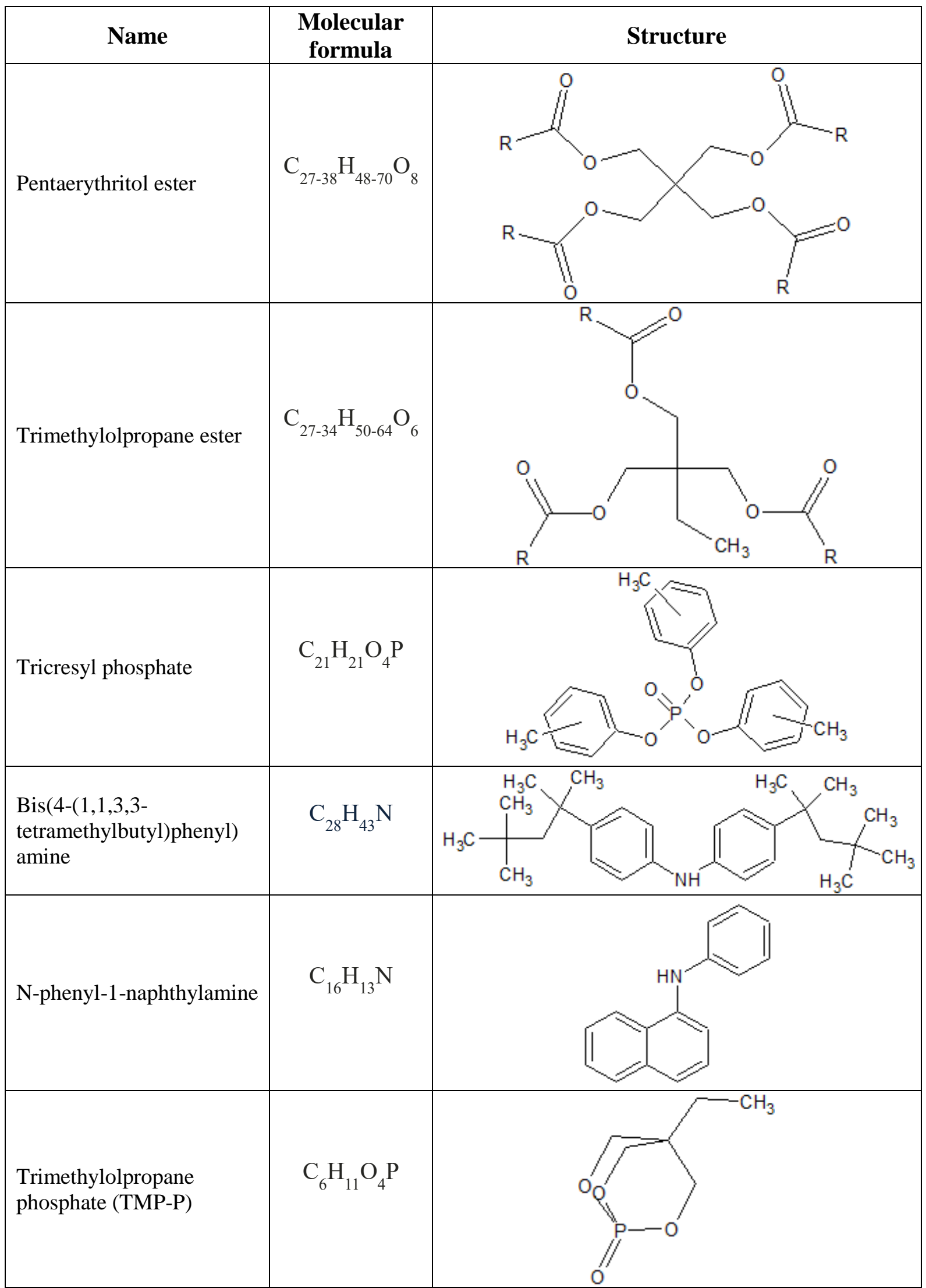




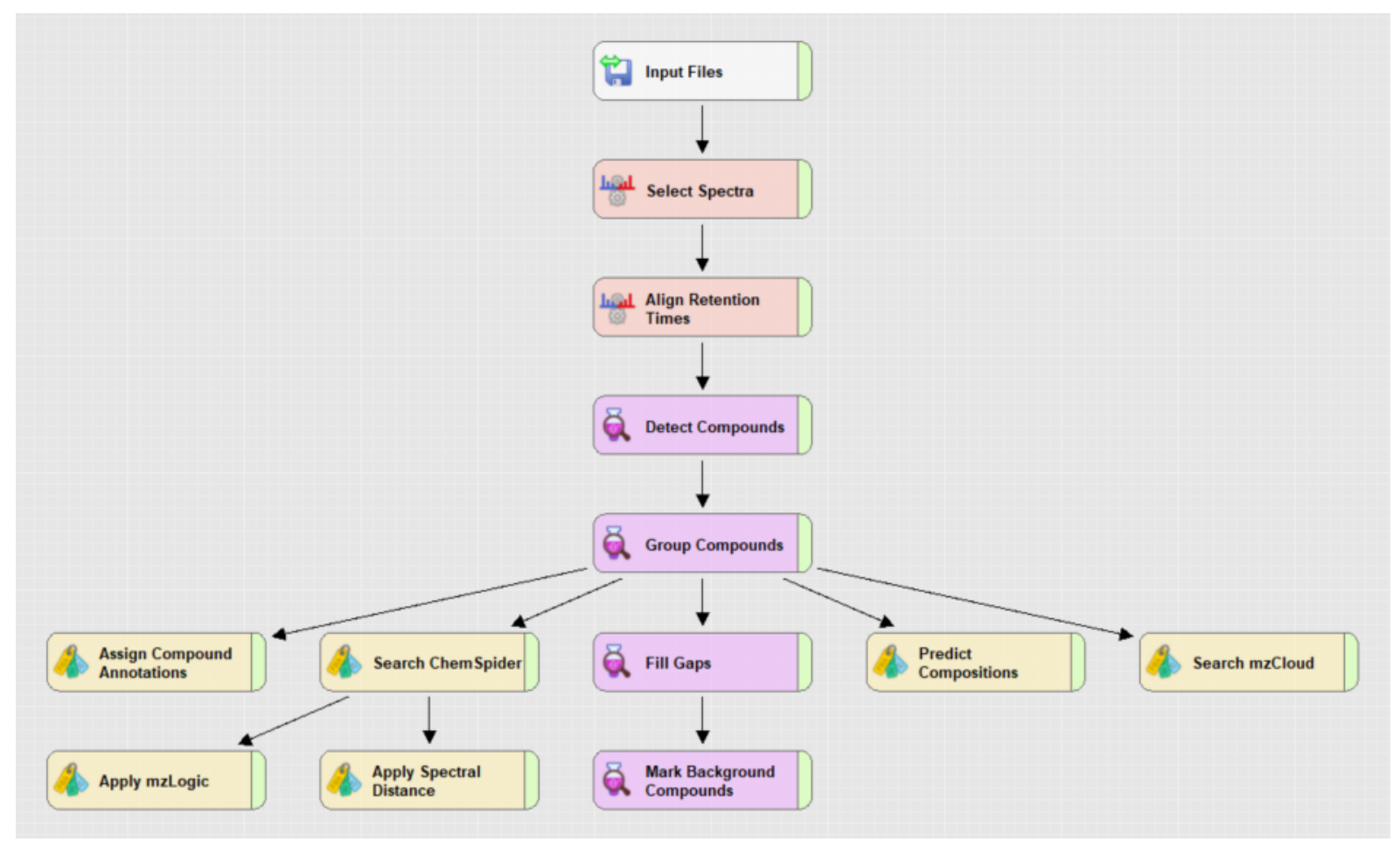

Figure S1. Workflow of the Compound Discoverer (CD) non-target screening. 
Table S3. Detailed settings of the CD-workflow.

Processing node 0: Input Files

Processing node 1: Select Spectra

1. Spectrum Properties Filter:

- Lower RT Limit: 0

- Upper RT Limit: 0

- First Scan: 0

- Last Scan: 0

- Ignore Specified Scans: (not specified)

- Lowest Charge State: 0

- Highest Charge State: 0

- Min. Precursor Mass: 0 Da

- Max. Precursor Mass: 5000 Da

- Total Intensity Threshold: 1000000

- Minimum Peak Count: 1

2. Scan Event Filters:

- Mass Analyzer: Is FTMS

- MS Order: Is MS2; MS1

- Activation Type: (not specified)

- Min. Collision Energy: 0

- Max. Collision Energy: 1000

- Scan Type: Any

- Polarity Mode: Is +

3. Peak Filters:

- S/N Threshold (FT-only): 1.5

4. Replacements for Unrecognized Properties:

- Unrecognized Charge Replacements: 1

- Unrecognized Mass Analyzer Replacements: ITMS

- Unrecognized MS Order Replacements: MS2

- Unrecognized Activation Type Replacements: CID

- Unrecognized Polarity Replacements: + 
- Unrecognized MS Resolution@200 Replacements: 60000

- Unrecognized MSn Resolution@200 Replacements: 30000

5. General Settings:

- Precursor Selection: Use MS1 Precursor

- Use Isotope Pattern in Precursor Reevaluation: True

- Provide Profile Spectra: Automatic

- Store Chromatograms: False

Processing node 2: Align Retention Times

1. General Settings:

- Alignment Model: Adaptive curve

- Alignment Fallback: Use Linear Model

- Maximum Shift [min]: 0.3

- Shift Reference File: True

- Mass Tolerance: 5 ppm

- Remove Outlier: True

Processing node 3: Detect Compounds

1. General Settings:

- Mass Tolerance [ppm]: 5 ppm

- Intensity Tolerance [\%]: 10

- S/N Threshold: 3

- Min. Peak Intensity: 500000

- Ions:

$[2 \mathrm{M}+\mathrm{H}]+1$

$[2 \mathrm{M}+\mathrm{K}]+1$

$[2 \mathrm{M}+\mathrm{Na}]+1$

$[2 \mathrm{M}+\mathrm{NH} 4]+1$

$[\mathrm{M}+\mathrm{H}]+1$

$[\mathrm{M}+\mathrm{H}+\mathrm{MeOH}]+1$

$[\mathrm{M}+\mathrm{K}]+1$

$[\mathrm{M}+\mathrm{Na}]+1$

[M+NH4]+1 
- Base Ions: $[\mathrm{M}+\mathrm{H}]+1 ;[\mathrm{M}+\mathrm{Na}]+1$

- Min. Element Counts: C H

- Max. Element Counts: C90 H190 Br3 Cl4 N4 O20 P S3

2. Peak Detection:

- Filter Peaks: True

- Max. Peak Width [min]: 0.5

- Remove Singlets: True

- Min. \# Scans per Peak: 5

- Min. \# Isotopes: 2

Processing node 5: Group Compounds

1. Compound Consolidation:

- Mass Tolerance: 2 ppm

- RT Tolerance [min]: 0.3

2. Fragment Data Selection:

- Preferred Ions: $[\mathrm{M}+\mathrm{H}]+1$

Processing node 6: Fill Gaps

1. General Settings:

- Mass Tolerance: 2 ppm

- S/N Threshold: 1.5

- Use Real Peak Detection: True

Processing node 7: Mark Background Compounds

1. General Settings:

- Max. Sample/Blank: 3

- Max. Blank/Sample: 0

- Hide Background: False

Processing node 9: Assign Compound Annotations

1. General Settings: 
- Mass Tolerance: 2 ppm

2. Data Sources:

- Data Source \#1：mzCloud Search

- Data Source \#2: Predicted Compositions

- Data Source \#3: (not specified)

- Data Source \#4: ChemSpider Search

- Data Source \#5: (not specified)

- Data Source \#6: (not specified)

- Data Source \#7: (not specified)

3. Scoring Rules:

- Use mzLogic: True

- Use Spectral Distance: True

- SFit Threshold: 20

- SFit Range: 20

Processing node 10: Search mzCloud

1. General Settings:

- Compound Classes: All

- Precursor Mass Tolerance: 5 ppm

- FT Fragment Mass Tolerance: 5 ppm

- IT Fragment Mass Tolerance: 0.4 Da

- Library: Autoprocessed; Reference

- Post Processing: Recalibrated

- Max. \# Results: 10

- Annotate Matching Fragments: False

2. DDA Search:

- Identity Search: HighChem HighRes

- Match Activation Type: True

- Match Activation Energy: Match with Tolerance

- Activation Energy Tolerance: 20

- Apply Intensity Threshold: True

- Similarity Search: Similarity Forward

- Match Factor Threshold: 60

3. DIA Search: 
- Use DIA Scans for Search: False

- Max. Isolation Width [Da]: 500

- Match Activation Type: False

- Match Activation Energy: Any

- Activation Energy Tolerance: 100

- Apply Intensity Threshold: False

- Match Factor Threshold: 20

Processing node 8: Predict Compositions

1. Prediction Settings:

- Mass Tolerance: 2 ppm

- Min. Element Counts: C H

- Max. Element Counts: C90 H190 Br3 Cl4 N4 O20 P S3

- Min. RDBE: 0

- Max. RDBE: 40

- Min. H/C: 0.1

- Max. H/C: 3.5

- Max. \# Candidates: 10

- Max. \# Internal Candidates: 200

2. Pattern Matching:

- Intensity Tolerance [\%]: 10

- Intensity Threshold [\%]: 0.1

- S/N Threshold: 3

- Min. Spectral Fit [\%]: 30

- Min. Pattern Cov. [\%]: 90

- Use Dynamic Recalibration: True

3. Fragments Matching:

- Use Fragments Matching: True

- Mass Tolerance: 5 ppm

- S/N Threshold: 3

Processing node 11: Search ChemSpider

1. Search Settings: 
- Database(s):

EAWAG Biocatalysis/Biodegradation Database

Nature Chemistry

Sigma-Aldrich

- Search Mode: By Formula Only

- Mass Tolerance: 5 ppm

- Max. \# of results per compound: 100

- Max. \# of Predicted Compositions to be searched per Compound: 3

- Result Order (for Max. \# of results per compound): Order By Reference Count (DESC)

2. Predicted Composition Annotation:

- Check All Predicted Compositions: False

Processing node 12: Apply mzLogic

1. Search Settings:

- FT Fragment Mass Tolerance: 10 ppm

- IT Fragment Mass Tolerance: $0.4 \mathrm{Da}$

- Max. \# Compounds: 0

- Max. \# mzCloud Similarity Results to consider per Compound: 10

- Match Factor Threshold: 30

Processing node 13: Apply Spectral Distance

1. Pattern Matching:

- Mass Tolerance: 5 ppm

- Intensity Tolerance [\%]: 30

- Intensity Threshold [\%]: 0.1

- S/N Threshold: 3

- Use Dynamic Recalibration: True 

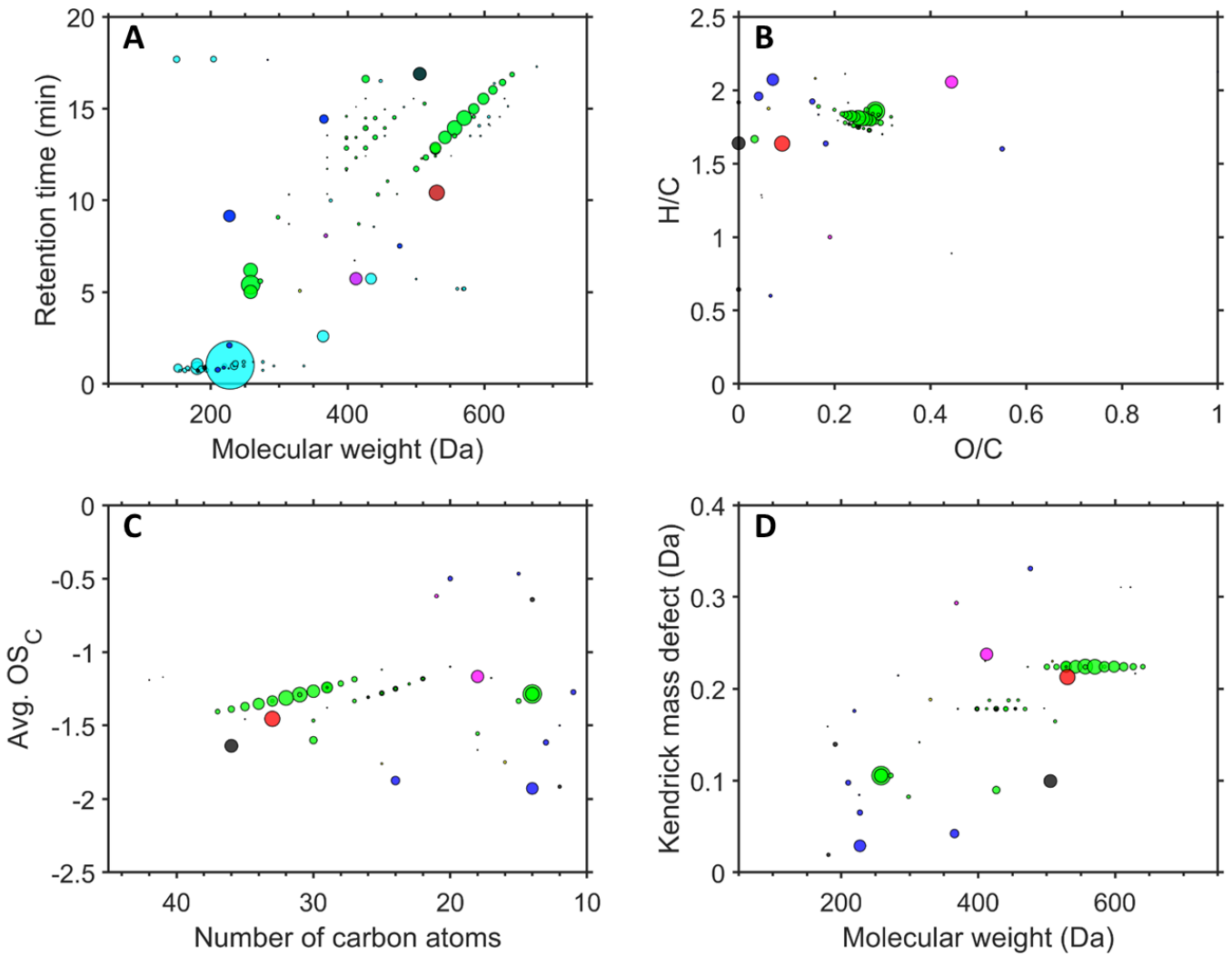

Figure S2. Molecular fingerprints (Retention time vs. MW [A], Van-Krevelen-diagram [B], Kroll-diagram [C], Kendrick mass defect vs. MW [D]) of eight averaged airport-related ultrafine particle samples in the size range of 0.010-0.018 $\mu \mathrm{m}$ (corrected). 

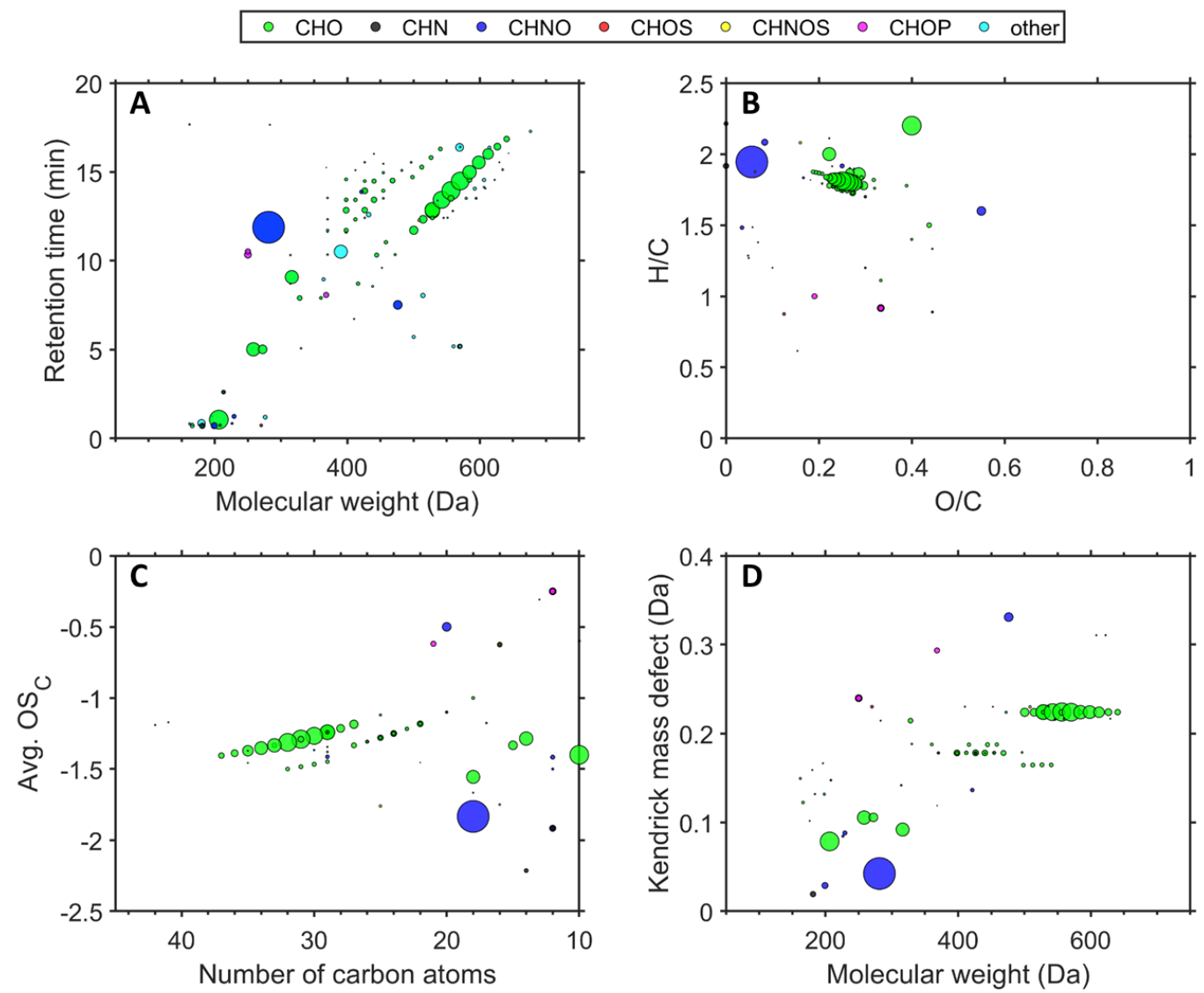

Figure S3. Molecular fingerprints (Retention time vs. MW [A], Van-Krevelen-diagram [B], Kroll-diagram [C], Kendrick mass defect vs. MW [D]) of eight averaged airport-related ultrafine particle samples in the size range of 0.018-0.032 $\mu \mathrm{m}$ (corrected). 

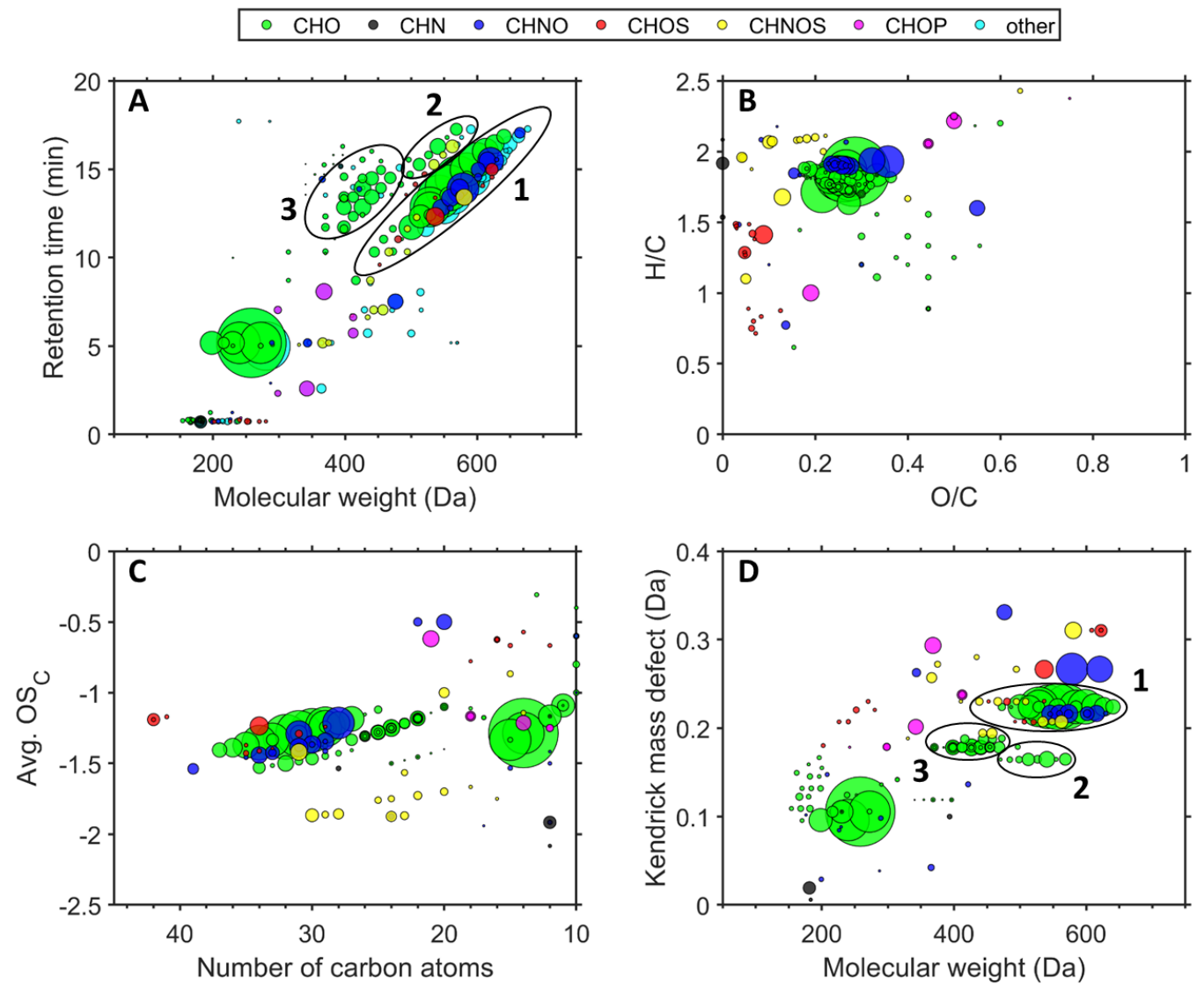

Figure S4. Native (not corrected) molecular fingerprints after non-target analysis (Retention time vs. MW [A], Van-Krevelen-diagram [B], Kroll-diagram [C], Kendrick mass defect vs. MW [D]) of six averaged airportrelated ultrafine particle samples in the size range of $0.032-0.056 \mu \mathrm{m}$ (for comparison with Figure 3). 


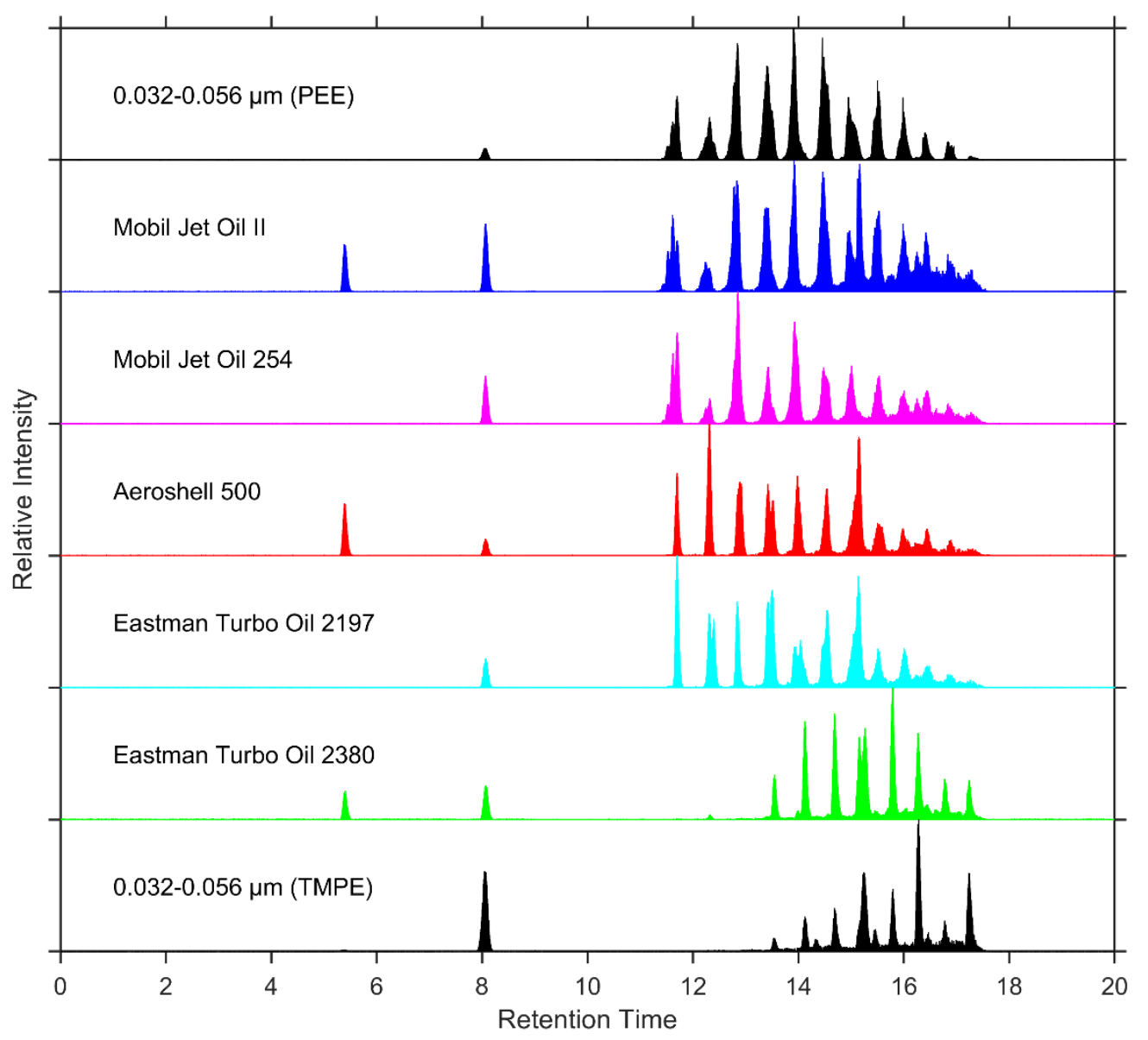

Figure S5. Extracted ion chromatograms (XIC) of an airport-sample (UFP size: $0.032-0.056 \mu$ m) and five different jet oils, showing a matching pattern of the major compounds $m / z \pm 4$ ppm 220.1120 (RT: $5.40 \mathrm{~min}$, $[\mathrm{M}+\mathrm{H}]^{+}$, N-phenyl-1-naphthylamine, $\left.\mathrm{C}_{16} \mathrm{H}_{13} \mathrm{~N}\right), \mathrm{m} / z \pm 4 \mathrm{ppm} 394.3468\left(\mathrm{RT}: 15.16 \mathrm{~min},[\mathrm{M}+\mathrm{H}]^{+}\right.$, alkylated diphenyl amine, $\left.\mathrm{C}_{28} \mathrm{H}_{43} \mathrm{~N}\right), \mathrm{m} / z \pm 4 \mathrm{ppm} 391.1069\left(\mathrm{RT}: 8.05 \mathrm{~min},[\mathrm{M}+\mathrm{Na}]^{+}\right.$, tricresyl phosphate, $\left.\mathrm{C}_{21} \mathrm{H}_{21} \mathrm{O}_{4} \mathrm{P}\right)$ and homologous series of $m / z \pm 4$ ppm 523.3241 - $677.4962\left(\mathrm{RT}: 11.70-17.27 \mathrm{~min},[\mathrm{M}+\mathrm{Na}]^{+}\right.$, pentaerythritol

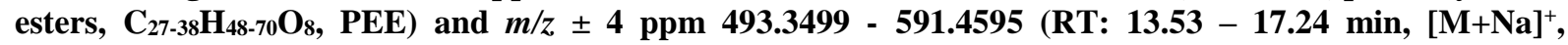

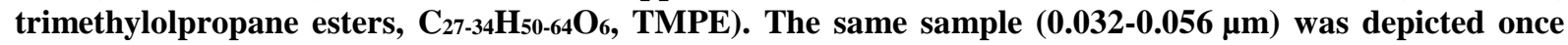
with the focus on the pentaerythritol esters (PEE) and once with the focus on the trimethylolpropane esters (TMPE) to illustrate the different influence of diverse jet engine lubrication oil base stock materials on ultrafine particles. 

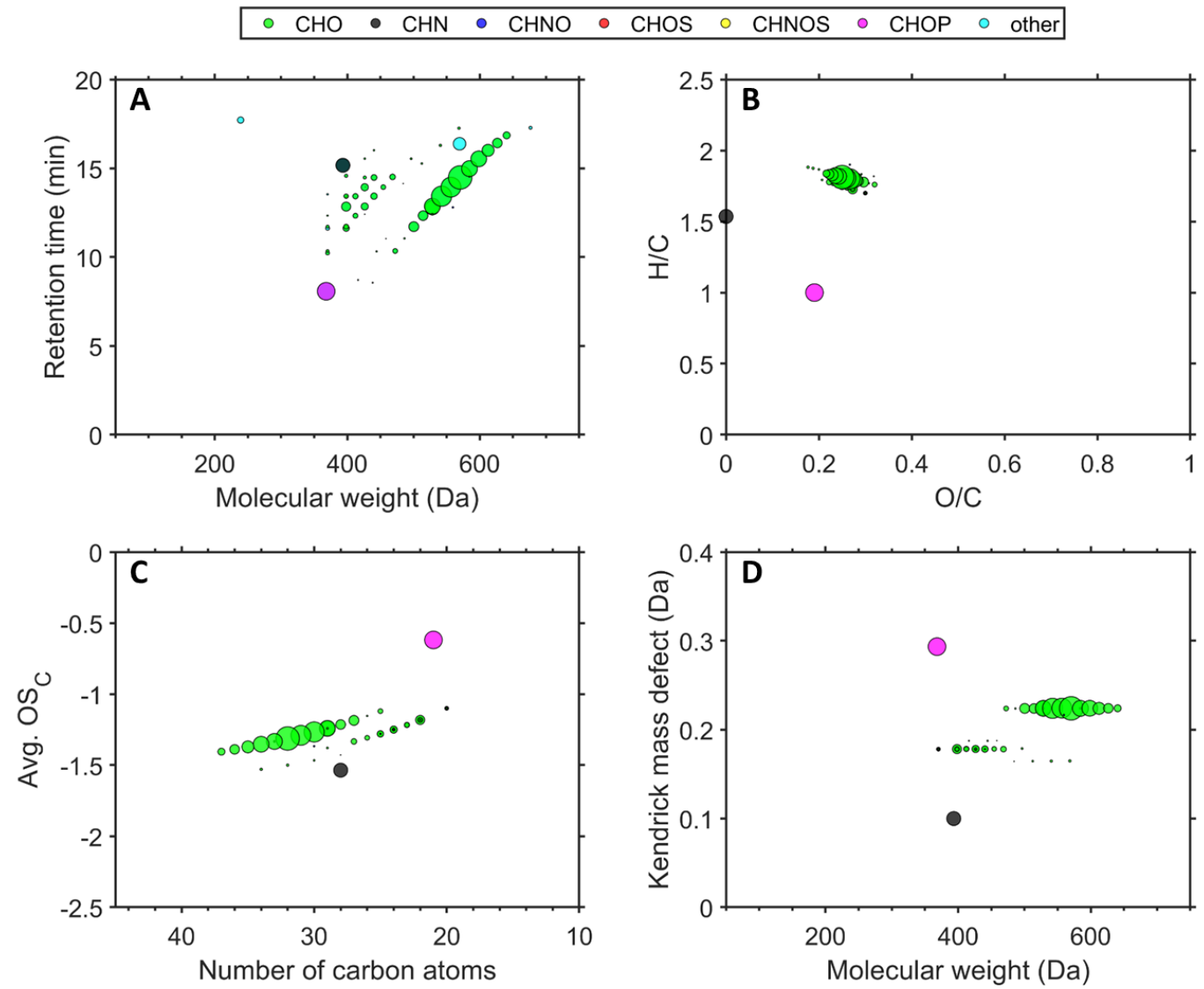

Figure S6. Molecular fingerprints (Retention time vs. MW [A], Van-Krevelen-diagram [B], Kroll-diagram [C], Kendrick mass defect vs. MW [D]) of the jet engine lubrication oil Mobil Jet Oil II by ExxonMobil. 

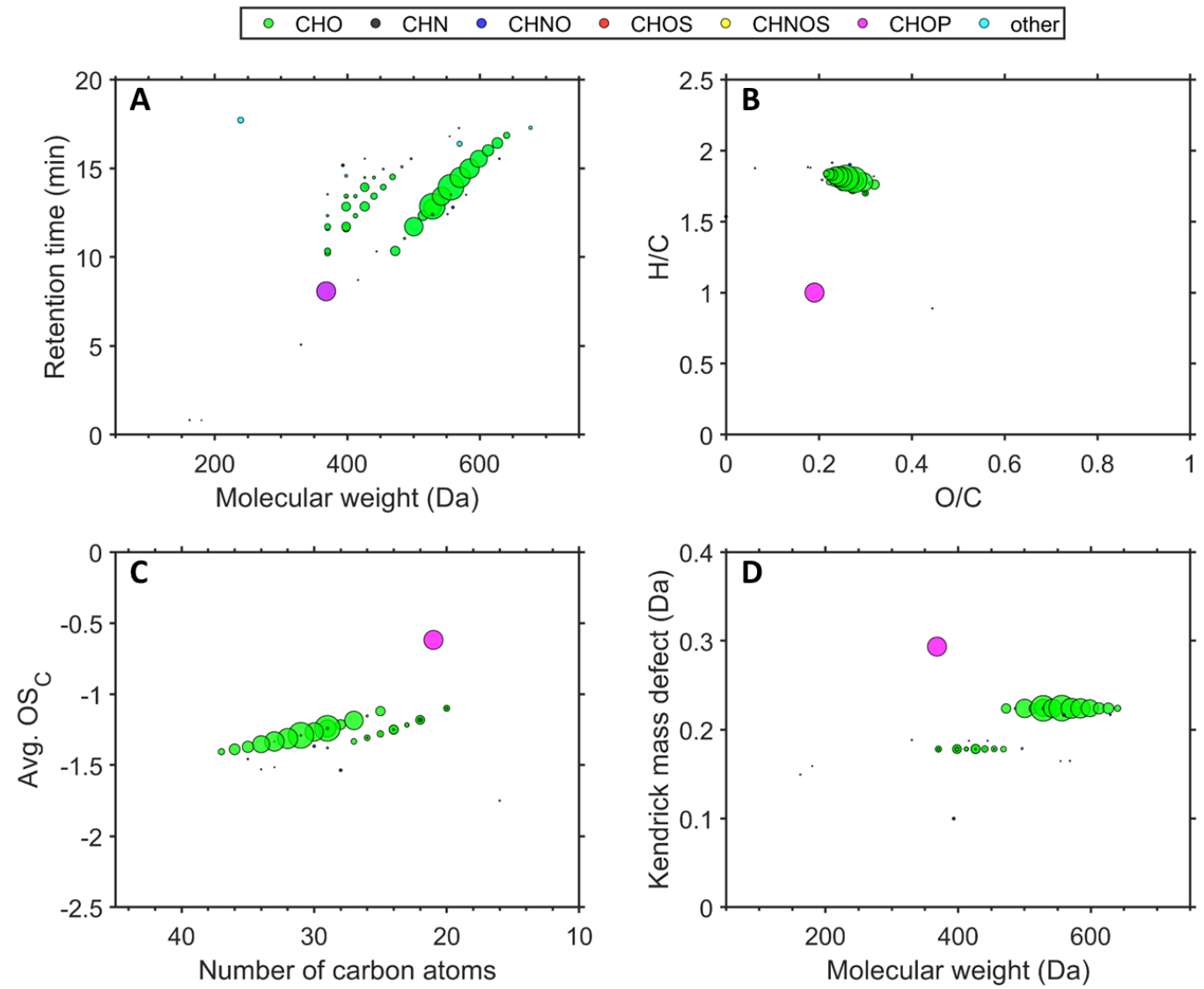

Figure S7. Molecular fingerprints (Retention time vs. MW [A], Van-Krevelen-diagram [B], Kroll-diagram [C], Kendrick mass defect vs. MW [D]) of the jet engine lubrication oil Mobil Jet Oil 254 by ExxonMobil. 

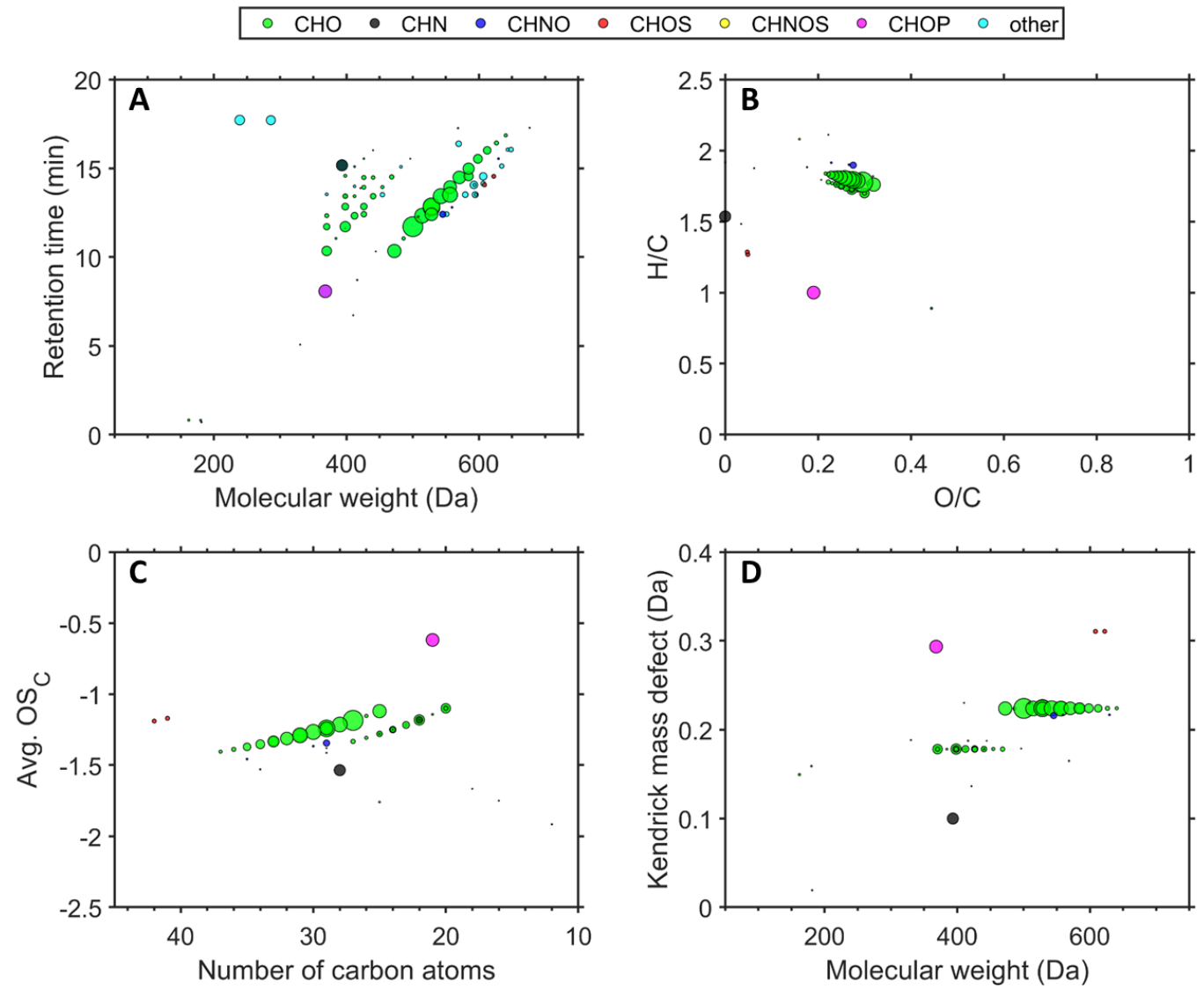

Figure S8. Molecular fingerprints (Retention time vs. MW [A], Van-Krevelen-diagram [B], Kroll-diagram [C], Kendrick mass defect vs. MW [D]) of the jet engine lubrication oil Turbo Oil 2197 by Eastman. 

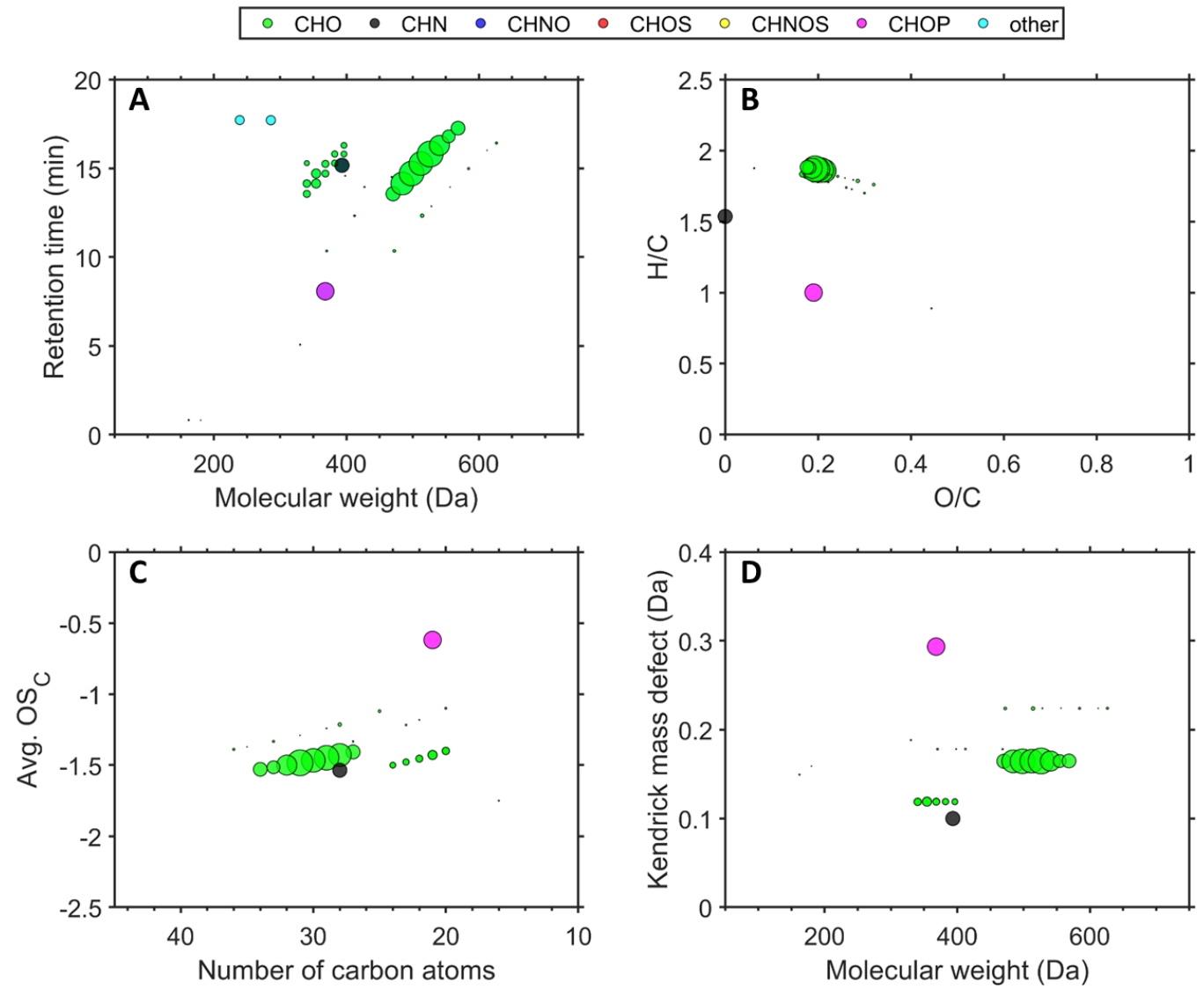

Figure S9. Molecular fingerprints (Retention time vs. MW [A], Van-Krevelen-diagram [B], Kroll-diagram [C], Kendrick mass defect vs. MW [D]) of the jet engine lubrication oil Turbo Oil 2380 by Eastman. 

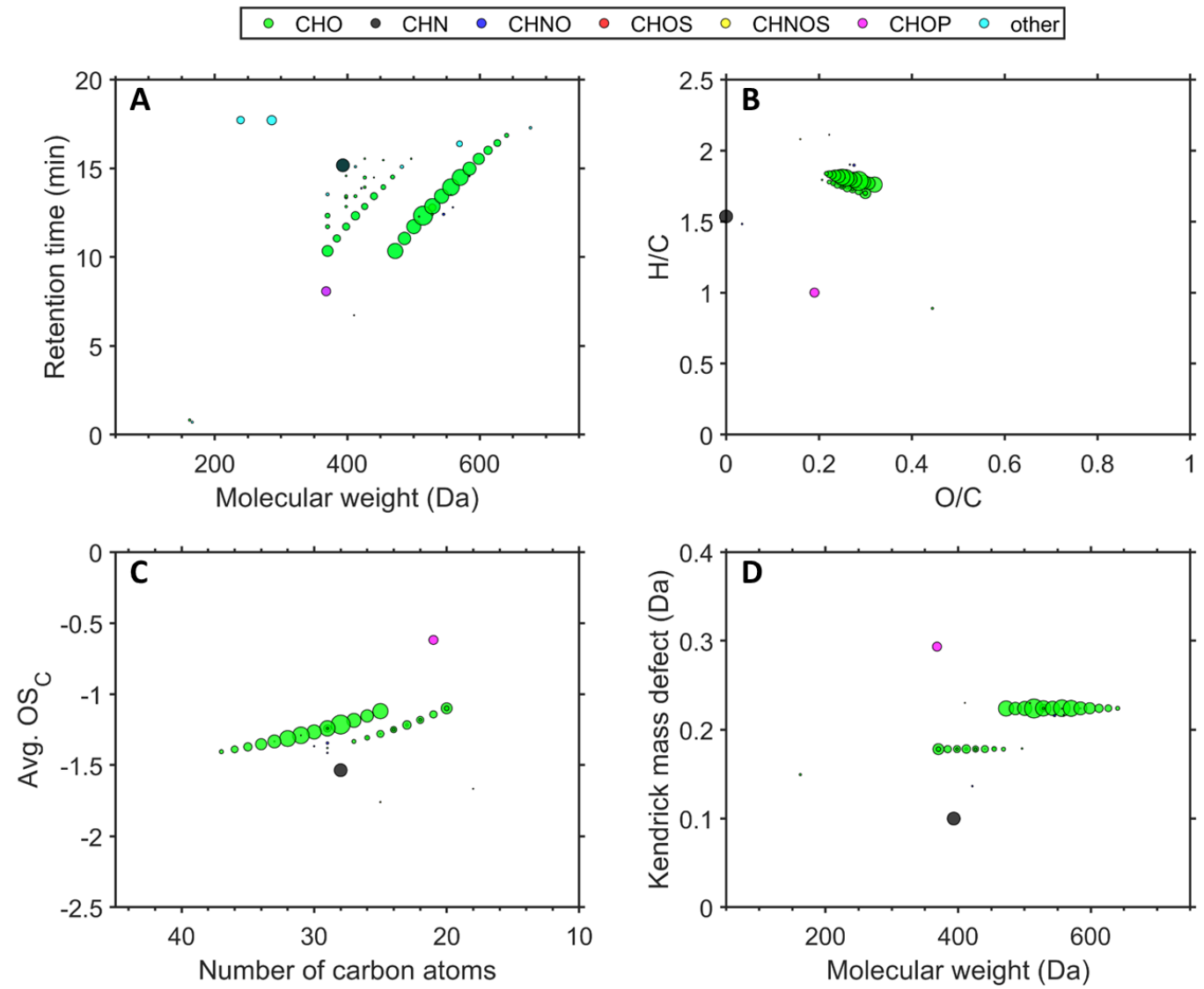

Figure S10. Molecular fingerprints (Retention time vs. MW [A], Van-Krevelen-diagram [B], Kroll-diagram [C], Kendrick mass defect vs. MW [D]) of the jet engine lubrication oil Aeroshell 500 by Royal Dutch Shell. 